\title{
Spondylolytic spondylolisthesis: various imaging features and natural courses
}

\author{
Tetsuo Nakayama $\cdot$ Shigeru Ehara
}

Received: 2 August 2014 / Accepted: 3 November 2014 / Published online: 23 November 2014

(C) Japan Radiological Society 2014

\begin{abstract}
The purpose of this paper is to review typical and atypical imaging features of spondylolytic spondyolisthesis and natural courses. Imaging features of the typical pars defect in early phase include bone marrow edema of the isthmus on MR imaging with progression of bone defect on plain radiography or CT. There are various defect types (pedicular cleft, retroisthmic cleft) and locations (upper lumbar spine and unilateral lesions). Natural courses also vary, with different patterns of progression and stabilization. Clinical correlation of the imaging and clinical features is often difficult.
\end{abstract}

Keywords Spondylolysis - Spondylolisthesis ·

Spondylolytic spondylolisthesis · Ithmic spondylolysis ·

Retroisthmic cleft $\cdot$ Pedicular (retrosomatic) cleft

\section{Introduction}

Spondylolytic spondylolisthesis is one of the major causes of low back pain in adolescents. Spondylolysis is defined as a bone defect of the posterior element of the vertebra. The isthmus (or pars interarticularis), which serves as a junction between the pedicle and the articular processes, is most commonly affected bilaterally. The fifth lumbar vertebra is involved in $95 \%$ of cases and the incidence decreases cephalad. Generally the defect is bridged by fibrous, cartilaginous or osseous tissue, and osseous fusion represents healing [1]. Spondylolisthesis is referred to as a displacement of the vertebra and there are two types, one associated

T. Nakayama $(\bowtie) \cdot$ S. Ehara

Department of Radiology, Iwate Medical University School of Medicine, Morioka 020-8505, Japan

e-mail: nakayama0110@yahoo.co.jp; ehara@iwate-med.ac.jp with spondylolysis and the other with degeneration of facet joints and/or intervertebral disc. Many patients with spondylolysis remain asymptomatic and certain cases become symptomatic, with low back pain and, less commonly, radicular pain. Spondylolisthesis can also be asymptomatic, but may cause low back pain, spinal instability, and radiculopathy, which requires conservative and/or surgical treatment.

The incidence of lumbar spondylolysis has been reported to be $8 \%$ in the adult population [2], and it was variable among races and sexes. Sakai et al. [3] reported that the prevalence of lumbar spondylolysis among the Japanese population between 20 and 29 years of age, found by multidetector CT, was $5.9 \%$, with a male to female ratio of 2:1. Spondylolysis has never been seen in newborn babies: it is seen only after walking. The upright posture and bipedal position play a role in the development of spondylolysis [4]. The prevalence of lumbar spondylolisthesis was reported to be $20.7 \%$ in the adult population, and spondylolysis without spondylolisthesis was relatively uncommon and was only seen at the lower lumbar level [5].

Although bone defects of typical isthmic spondylolysis run obliquely through the pars interarticularis, and are well depicted on oblique lumbar radiography, atypical isthmic defects have been reported in several papers [6, 7]. The pathomechanism and incidence of atypical isthmic defects is not well known, and the radiographic appearance of atypical isthmic spondylolysis has not been well recognized among radiologists. Other atypical spondylolyses included various types, and two variations have been reported in the literature [8-10]. Unilateral spondylolysis accounts for 3-33\% of all cases of spondylolysis and has been estimated to be approximately 3-7 \% of the general population [11]. It may be associated with certain athletic activities, such as cricket, ballet, and gymnastics, and the association with 
Table 1 Classification of spondylolysis modified by Wiltse

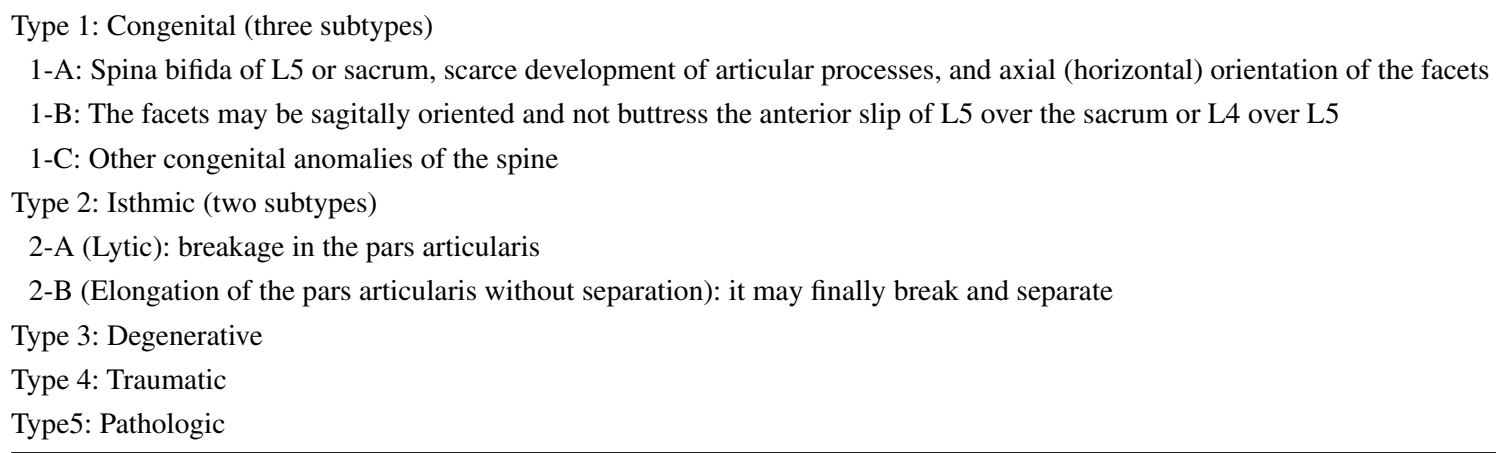

Table 2 Classification system by Marchetti and Bartolozzi

\begin{tabular}{l}
\hline Primary category: Acquired \\
Traumatic \\
Acute fracture \\
Stress fracture \\
Postsurgical \\
Direct \\
Indirect \\
Pathologic \\
Local \\
Systemic \\
Degenerative \\
Primary \\
Secondary \\
Primary category: Developmental \\
High dysplastic \\
With lyses \\
With elongation \\
Low dysplastic \\
With lyses \\
With elongation \\
\hline
\end{tabular}

facet tropism has been investigated [12]. The involvement of the upper lumbar spine has been rarely noted, and clinical aspects and radiological features of spondylolysis of the lumbar spine have been reported in the literature [7, 13-15].

The aim of this article is to present various imaging features of spondylolitic spondylolisthesis, including early and late changes of typical isthmic spondylolysis and atypical types of spondylolysis and/or spondylolitic spondylolisthesis in addition to their clinical courses.

\section{Classification}

The classification of spondylolisthesis proposed by Newman and Wilste [16] has been accepted widely, and it was modified in 1990. According to this classification, spondylolistheses are divided into six types (Table 1). Another classification system of spondylolisthesis was developed by Marchetti and Bartolozzi [17] (Table 2). This classification includes two main categories: one is developmental deficiencies at the lumbosacral junction; and the other is acquired, including traumatic, iatrogenic, pathologic, and degenerative changes. The main difference between these two classification systems is that Marchetti and Bartolozzi's system avoids the term "isthmic" and emphasizes the distinction between developmental dysplastic slips and acquired stress fractures of the isthmus. Marchetti and Bartolozzi's classification system is thought to be applicable to all forms of lumbar spondylolisthesis [18], but it has not been recognized as the standard classification.

\section{Etiology}

The pathogenesis of spondylolisthesis is still unclear, and two theories, traumatic and congenital, are known [19]. The traumatic theory is based on a recent history of trauma in a patient, and high incidence of isthmic lyses in athletes, such as gymnasts and weight lifters. Ward et al. described an inadequate increase in the transverse interfacetal distance progressing caudally from L4 to S1, compared with normal control subjects, was found in the individuals with spondylolysis of L5. The inferior articular process of L4 and the superior articular process of S1 contact the same cross-sectional position of the L5 pars interarticularis in accordance with the reduction in the transverse interfacetal distance, so excessive pressure was applied on the isthmus due to pinching between the inferior articular process of the segment above and the superior articular process of the segment below, thus leading to bony resorption and bone weakness, finally resulting in lysis [20]. In the analysis of cadaveric skeletons with L5 spondylolysis, Masharawi et al. noted that greater degrees of coronal orientation, facet tropism and symmetry of the lower lumbar facets was 
strongly associated with spondylolysis, and with increased contact surface area of the opposing articular processes because of coronally oriented facet joints: they postulated that more loading stress was being applied to the isthmus during flexion and extension.

In the congenital or developmental theory, the lysis is thought to be the result of the normal mechanical forces of the erect position toward the dysplastic isthmus and surrounding bone structures [21]. This is also supported by the high incidence of spondylolisthesis observed in certain ethnic groups and the heredity of spondylolisthesis in some families. It might be reasonable that repetitive activities or micro trauma to the congenitally weak isthmus and surrounding bone lesion leads to the pars lysis [22]. Disruption of the posterior elements caused by isthmic lysis or congenital dysplasia can weaken their tension-band effect and lead to anterior migration of the vertebral body, resulting in spondylolisthesis [23]. Isthmic spondylolysis can diminish the stability of the posterior elements of the lumbar vertebrae and lead to spondylolisthesis during adulthood [24]. Mihara et al. [25] described bilateral spondylolyses as increasing the intervertebral mobility at the involved vertebral segment and at the vertebral segment above the involved segment. This result supports the pathomechanism of spondylolysis development to spondylolisthesis. The progression of spondylolysis to spondylolisthesis most commonly occurs during adolescents younger than 16 years of age, and decreases with advanced age [26].

The mechanism of the slippage of the lumbar spine in skeletally immature subjects is still being debated. Farfan et al. documented the growth zone near to the vertebral endplate: the intervertebral disk was the weakest portion during growth, and pediatric spondylolisthesis may occur after epiphyseal separation [27]. Sairyo et al. proposed a pathomechanism for pediatric spondylolisthesis. Their pathomechanism was as follows: spondylolysis changes the spinal kinematics, leading to stress concentration at the growth plate during motion, and continuous stress loading to the growth plate can lead to fracture at the vertebral body, resulting in spondylolisthesis [28]. Spino-pelvic parameters, including the sacral slope, pelvic tilt, and pelvic incidence, measured by lateral radiographs of the lumbosacral spine, may also have an important role in the development of spondylolisthesis due to spondylolysis. A high pelvic incidence is a predisposing factor for L5 spondylolisthesis because high pelvic incidence indicates a high sacral slope and/or pelvic tilt, and may increase lumbar lordosis, which contributes to high shear stress to the L5 isthmus, resulting in lysis [1].

In adult patients, it is widely accepted that the development of vertebral slippage in the lumbar spine is related to disc degeneration, which may cause conversion from stable spondylolisthesis to unstable vertebral spondylolisthesis
[29]. It was demonstrated by the studies of McGregor et al. and Axelsson et al. [30-32] that a spondylolysis does not contribute to progression of instability or hypermobility of the lumbar spine, or increased progression of disc degeneration below the level of the spondylolytic vertebra.

\section{Isthmic spondylolyses}

\section{Typical isthmic spondylolyses}

The typical isthmic spondylolysis presents as a radiolucent defect of the pars articularis (Fig. 1). A typical defect runs obliquely through the isthmus and is well documented on the oblique and lateral views. The pathogenesis is considered to be a stress fracture. Fracture of the isthmus begins at the inferior or inferomedial portion of the isthmus and propagates superiorly or superolaterally; whereas fracture healing occurs in the reverse direction [33]. The early stage of isthmic spondylolysis is very difficult to diagnose by radiography or $\mathrm{CT}$ and, before the cortical breakage of the isthmus, stress reaction of bone marrow due to repetitive mechanical loading is only seen on MR imaging. The fluidsensitive sequences, such as fat-suppressed T2-weighted and STIR images, are sensitive to such bone marrow edema.

With progression of spondylolyses, fracture of the isthmus can be recognized as a linear low signal intensity on T1- and T2-weighted images, surrounded by an area of bone marrow edema. The marginal sclerosis showing low signal intensity on both $\mathrm{T} 1$ - and $\mathrm{T} 2$-weighted images may represent disease progression. Fracture healing may not occur before bone marrow edema [34]. As fracture healing progresses, the fracture line disappears with marginal sclerosis. The signal intensity of the defect is variable: low on T1-weighted images, low to intermediate on T2-weighted images when the defect is composed of fibrous or fibrocartilaginous tissue, and low on both T1- and T2-weighted images when osseous union is noted. In the case of nonunion, fibrous scar and/or fluid may be seen in the gap. The signal intensity of the gap may be inhomogeneous as the result of the mixed fibrous liquid components [1].

Visualization of incomplete stress fracture of the isthmus is still challenging for MR imaging. Dunn et al. [33] reported that MR imaging was limited for depicting the cortical integrity of incomplete fractures, and all incomplete fractures showed as a breakage in the inferomedial portion of the isthmus on CT. Several grading systems for isthmic spondylolsis based on MR imaging have been widely used: Hollenberg et al. [35, 36] proposed a classification system for MR imaging, with high intraobserver and interobserver reliability, as well as correlating well with CT and SPECT (Table 3). 

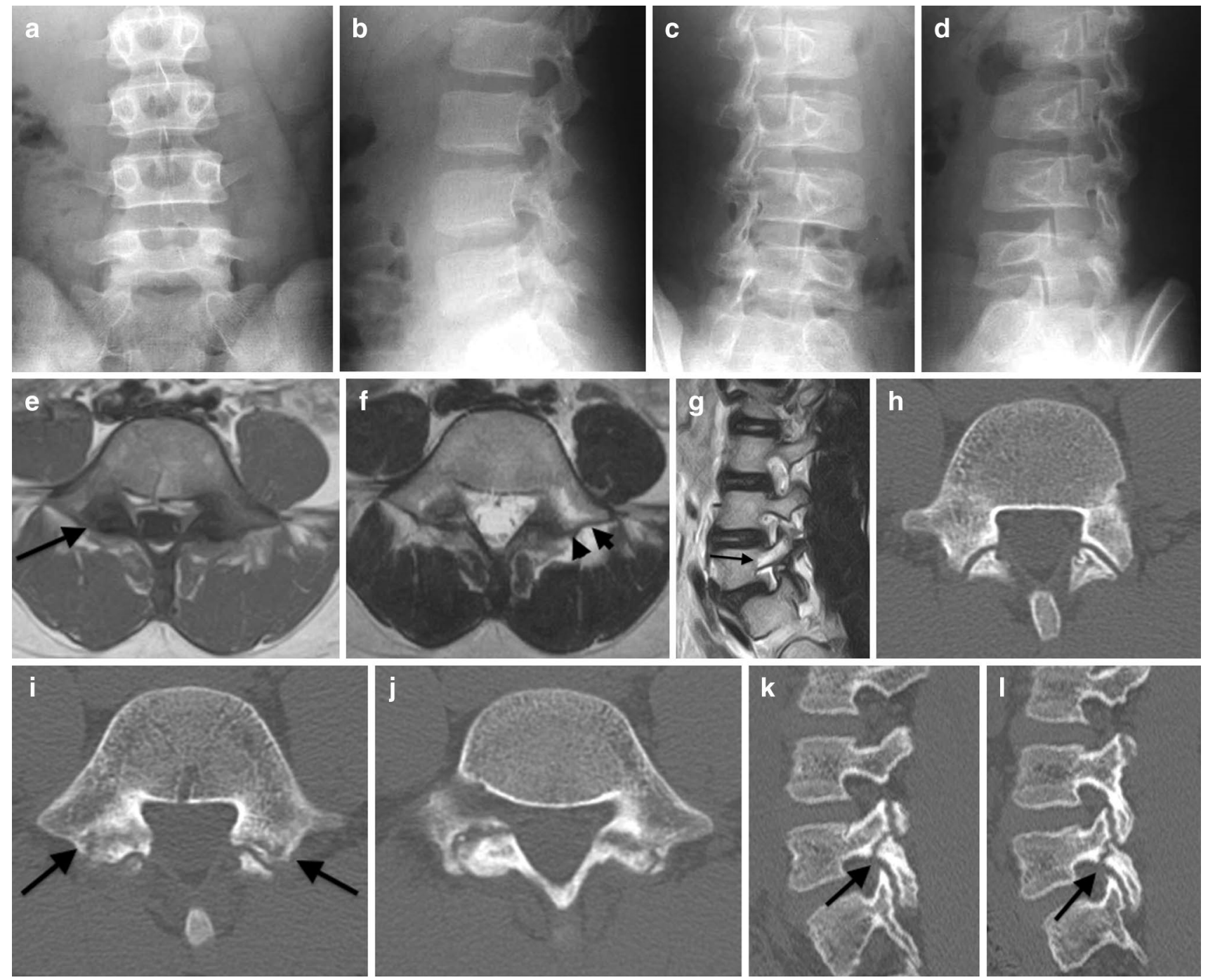

Fig. 1 Typical isthmic spondyolysis (12-year-old male with back pain). Plain radiography of the lumbar spine. a AP view, b lateral view, $\mathbf{c}$ left posterior oblique view, $\mathbf{d}$ right posterior oblique view. No bone abnormality is seen in MRI of L5 level. e Axial T1-weighted image, $\mathbf{f}$ axial T2-weighted image. A linear low signal lesion extending through right pars is seen on T1-weighted image (arrow). An inhomogeneous high signal lesion in both pedicle and pars on T2-weighted image is also noted, and it shows bone marrow edema (arrow). Patchy low-signal lesion adjacent to the lysis showing reac-

tive sclerosis also noted (arrow). g T2-weighted right parasagittal image. Bone marrow edema is clearly shown and the lysis of the pars is also demonstrated as a short linear high-signal lesion (arrow). CT of the L5 level obtained 3 months later. $\mathbf{h}, \mathbf{i}, \mathbf{j}$ Serial axial image from cranial to caudal. The defects of the bilateral pars are demonstrated with surrounding sclerosis, and the margin of both clefts is irregular (arrows). k, I Parasagittal multiplanar reconstruction through the isthmus. The cleft of both pars runs obliquely (arrows)

Table 3 MR imaging classification of spondylolysis by Hollenberg et al. (from Ref. [36])

Grade 0: Normal marrow signal with intact cortical margin of the isthmus

Grade 1: Bone marrow edema with intact cortical margin of the isthmus with or without signal changes in adjacent pedicle or articular processes Grade 2: Incomplete fracture showing bone marrow edema and cortical breakage incompletely running though isthmus

Grade 3: Complete fracture denoting a complete bone defect of isthmus with bone marrow edema

Grade 4: Fracture non-union presenting complete separation of isthmus without bone marrow edema

MR diagnosis of spondylolysis without spondylolisthesis can be difficult without ancillary findings. Ulmer et al. [37] found that a widened sagittal diameter of the spinal canal, wedging of the posterior aspect of the vertebral body at the level of spondylolysis, and bone marrow signal changes of the pedicle adjacent to the isthmic spondylolysis 

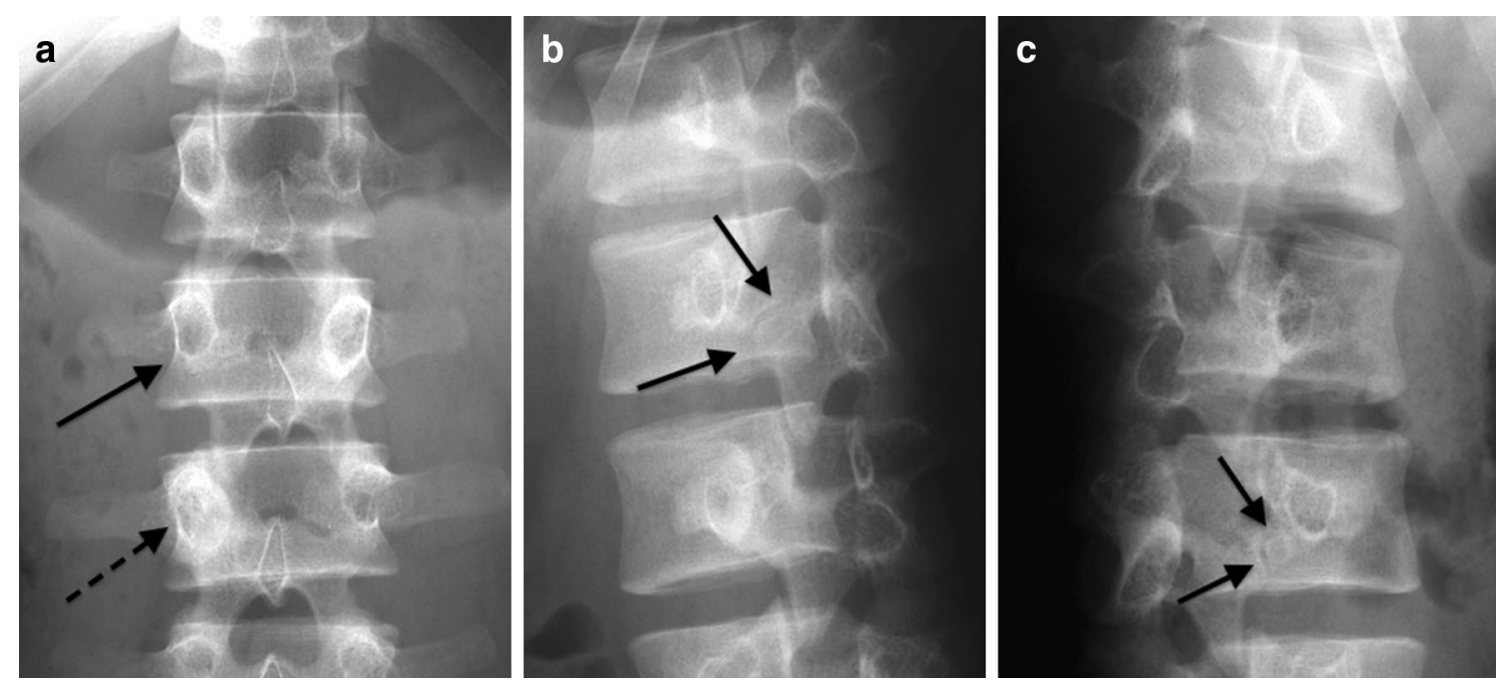

Fig. 2 Atypical isthmic spondylolysis (28-year-old male with back pain). Plain radiography of the lumbar spine; a AP view: $\mathbf{b}$ right posterior oblique view: c left posterior oblique view. a A defect extending transversally is seen below the right pedicle at L2 (arrow). The right pedicle of L3 is enlarged with sclerosis (dotted arrow), b the

were useful in making a diagnosis of lumbar spondylolysis without or with spondylolisthesis. Epidural fat interposition between the dura mater and the L5 spinous process is known as an indirect sign on midsagittal MR images of the lumbar spine [38].

CT is believed to be the most useful modality in the evaluation of size and location of the isthmic fracture. Fujii et al. described the fracture of the isthmus as having three stages: early, progressive, and terminal [39]. The early stage showed a narrow fissure through the isthmus with a sharp margin. The progressive stage was defined as a narrow fissure with a slightly rounded margin. The terminal stage had a wide and round margin and sclerosis. They also noted that a significantly greater proportion of defects achieved union in the early stage compared with progressive and terminal stages, and no bony union was seen in the terminal stage [39].

\section{Atypical isthmic spondylolysis}

Although typical isthmic spondylolysis that runs obliquely through the pars interarticularis is commonly seen in the lumbar vertebra, isthmic spondylolysis with a different direction through the pars interarticulars is known (Fig. 2). Nathan classified spondylolysis into typical and atypical types according to the shape and course of the bone defect [6]. Among atypical spondylolyses, one type has a vertical line at the inferolateral part (the lower border of the isthmus) and a horizontal line at the superomedial part (through the lamina). Another type has a vertical superomedial portion running from the inferior edge of the superior defect has a superomedial portion running horizontally (arrow) and an inferolateral portion running vertically (arrow). c The defect, which has an atypical course in the left pars of L3 (arrows), is also seen

articular surface and is horizontal at the inferolateral portion reaching the lateral margin of the lamina.

The prevalence of atypical defects of pars is uncertain. Lee et al. [7] found nine atypical defects among 25 defects of the three upper lumbar vertebrae, and five atypical defects among 27 defects of pars from T12 to L6 were seen in the 29 vertebrae of an adult skeleton study [15]. Lee et al. also reported the defect having the vertical inferolateral portion, and the horizontal superomedial portion was seen in the majority of the patients with atypical spondylolyses. These clefts were not found in the lumbar vertebrae of the 700 dissected fetuses and neonates in Wiltses' study [40] and 500 neonates radiographed in Fredrickson's study [26]. It is suggested that atypical isthmic spondylolysis is not congenital but multifactorial, and that a pre-existing bony discontinuity in the superomedial or the inferolateral portion may lead to mechanical weakness of posterior elements of vertebrae and cortical breakage of the other site may occur by repeated shearing forces to the facets during extension and flexion.

\section{Non-isthmic spondylolyses}

Non-isthmic spondylolyses include various types, and two variations have been reported in the literature [10, 41, 42]. Clefts in various locations of the lamina have been known, and six locations exist, including the retroisthmic and pedicular clefts (Fig. 3). It has been postulated that these cleft are congenital in origin, and they are considered to be the result of stress fractures [42, 43]. Based on recent 


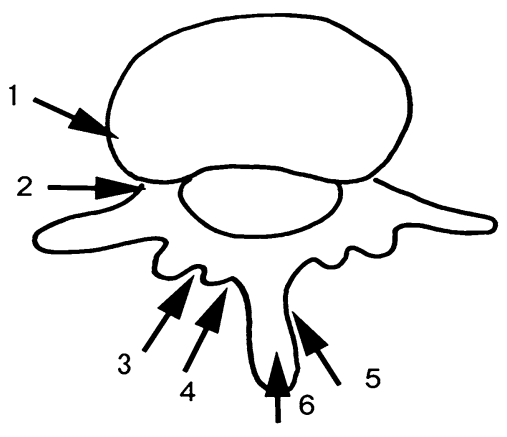

Fig. 3 Clefts in the vertebral arch. 1 Neurocentral synchondrosis, 2 pedicular (retrosomatic) cleft, 3 pars interarticularis (isthmic) cleft, 4 retroisthmic cleft, 5 paraspinous cleft, 6 spinous cleft (spina bifida)

studies, it is postulated that the orientation and shape of the facet joints, such as more frontally oriented facet with a wide surface during extension and flexion and higher stresses on the posterior element, is related to the development of these clefts $[12,21,44]$.

\section{Retroisthmic cleft}

The retroisthmic cleft is a bone defect in the lamina immediately dorsal to the inferior articular process (Fig. 4). The term laminolysis is also used. L5 is the most frequent location, followed by L4. Distribution of the defect in L3 and above has not been observed. This distribution is similar to that of pars spondylolysis [42]. In the reported cases, hypoplastic, ipsilateral pedicle and unilateral or contralateral spondylolysis were associated with a retroisthmic cleft [45-47]. Diagnosis of a retroisthmic cleft is made by finding a cleft running vertically though the lamina. Oblique radiography and $\mathrm{CT}$ are sensitive in detecting a cleft in the lamina. An MR imaging feature of the retroisthmic cleft has been reported in a few case reports, showing as a linear high-signal lesion on T2-weighted images [48]. The natural course is not well understood. In a case followed over 6 years with conservative treatment, slow progression of a cleft with a sclerotic margin has been reported [42]. They concluded that the retroisthmic cleft was the result of a stress fracture of the lamina underlying some congenital anomaly in the posterior element.

Pedicular (retrosomatic) cleft

Diagnosis of a pedicular cleft is easily made by finding a lysis beneath the pedicle on oblique radiography (Fig. 5). In the reported cases with $\mathrm{CT}$ and MR imaging, the pedicular cleft was surrounded by sclerosis of adjacent bone which consisted of chronic pedicle stress reaction and early spondyloytic change [41, 43]. The incidence is uncertain. In reported cases, contralateral [10], or unilateral spondylolysis [49] were associated with a pedicular cleft, and a case of a pedicular cleft with abnormal morphology due to neurofibromatosis has also been described [50]. A congenital or developmental anomaly of the affected vertebrae may be related to the formation of the pedicular clefts [41]. A stress fracture has also been considered to be the cause. Previously reported cases of pedicular clefts in athletes, such as weight lifters, soccer, baseball, and volleyball players, ballet dancers and gymnasts, whose repetitive shearing and rotational forces were loaded to the pedicle, have been documented $[10,43,51]$. Sairyo et al. showed, using finite element analysis, that cumulative stresses across the contralateral neural arch can lead to pedicular fracture and progress to pediculolysis [43]. Pedicular fractures in patients with degenerative lumbar spondylosis, osteoporosis due to medication with predonisone, and after spinal surgery have also been noted and reported to occur unilaterally and bilaterally $[41,52,53]$.

\section{Other atypical distributions}

\section{Unilateral spondylolysis}

Unilateral spondylolysis is considered to be rare, and it accounts for 3-33\% of all cases of spondylolysis [54].
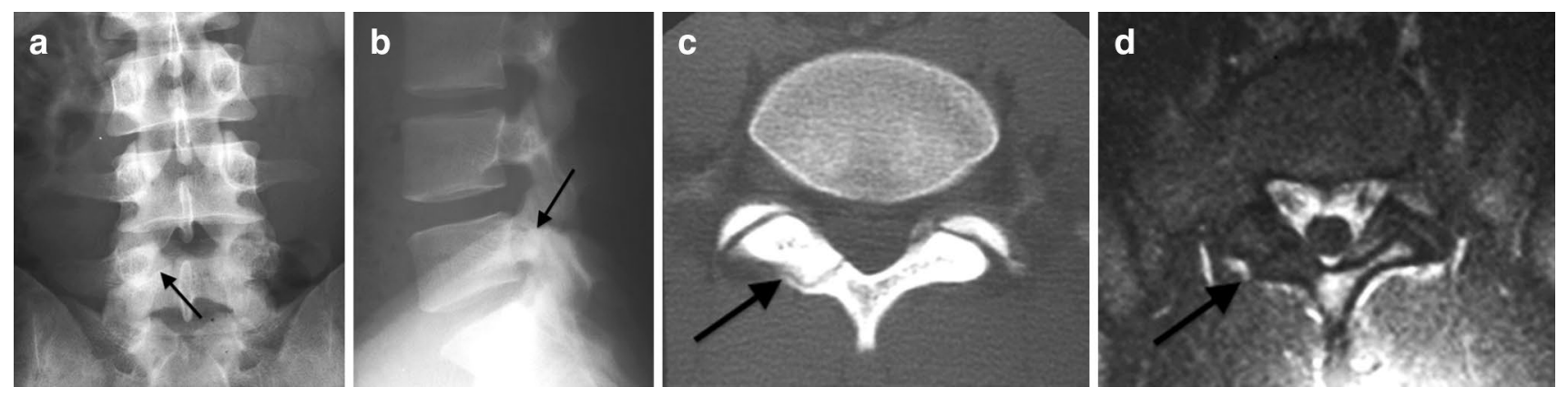

Fig. 4 Retroisthmic cleft (19-year-old male with back pain). Plain radiography; a AP view, b lateral view. a, b A defect in the right pedicle runs obliquely (arrow). Marginal sclerosis is noted (arrow), c CT of L5. The defect of the right lamina with surrounding sclerosis is evident (arrow). d T1-weighted axial image of L5. The defect is seen as a low-signal band (arrow) 

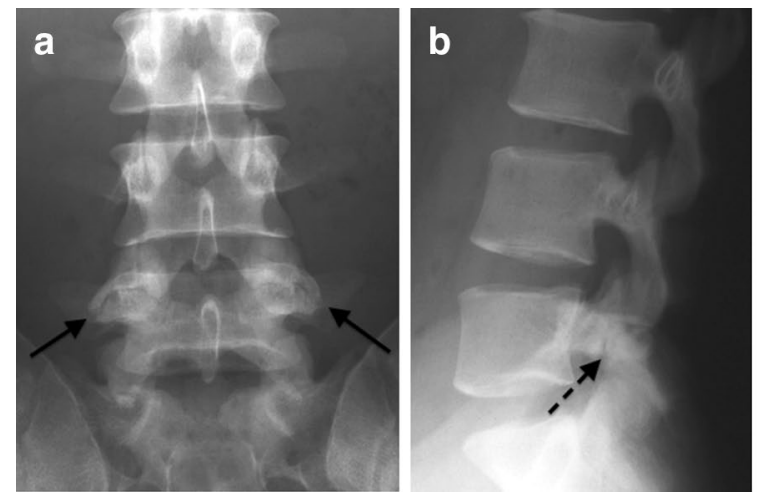

Fig. 5 Pedicular cleft (23-year-old male with back pain). Plain radiography of the lumbar spine. a AP view b lateral view, c right posterior oblique view, $\mathbf{d}$ left posterior oblique view. a, b Defects in the
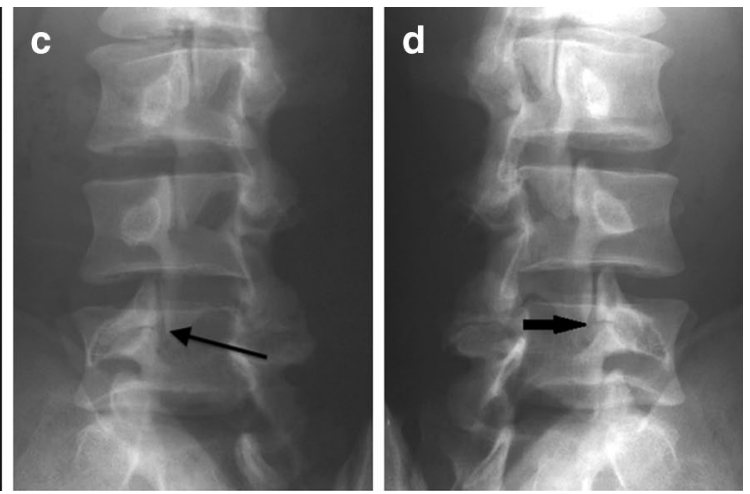

bilateral pedicles are documented without slippage (a arrows, b dotted arrow). c, d Both defects extend transversally through the pedicles (arrows)
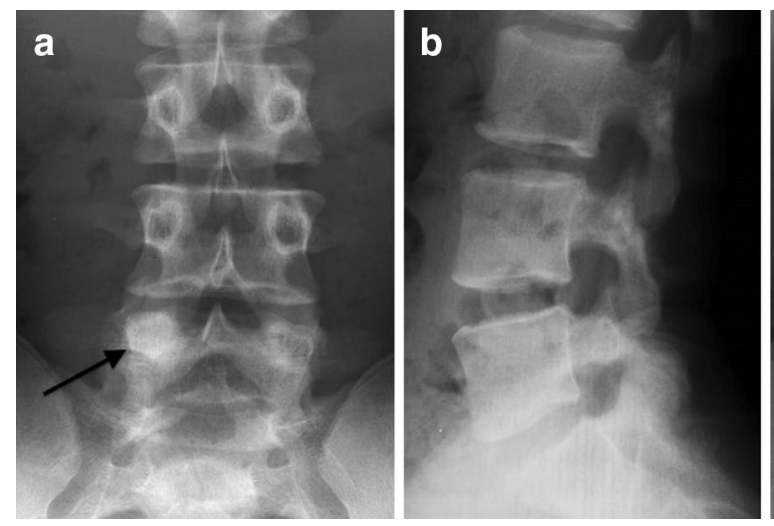

Fig. 6 Unilateral spondylolysis (14-year-old male with back pain). Plain radiography of the lumbar spine; a AP view: b lateral view: c right posterior oblique view: $\mathbf{d}$ left posterior oblique view. a Sclerosis of the right pedicle of L5 is seen on AP view. This appearance is the

Patients with unilateral spondylolysis can be symptomatic or asymptomatic. The level involved can be variable, but it is common in lower lumbar vertebrae. The pathogenesis is controversial. A congenital defect of unilateral pars is a possible cause. Rankine et al. have postulated that unilateral spondylolysis may occur on the side of the more coronally oriented facet joint because of asymmetric force though the contralateral side [12]. Atypical clefts are considered to be frequent [7]. There are many indirect signs of unilateral spondylolysis such as contour bulging of the involved pars interarticularis, reactive sclerosis of the contralateral pedicle, asymmetric neural arch, deviation or step-off of the spinous process, and widening of the spinal canal with anterolisthesis or posterior subluxation of the posterior element (Fig. 6) [11, 37]. Sclerosis of the contralateral pedicle showing the "dense pedicle sign" should be differentiated from other diseases, such as osteoid osteoma and Paget's disease. Spondylolisthesis associated
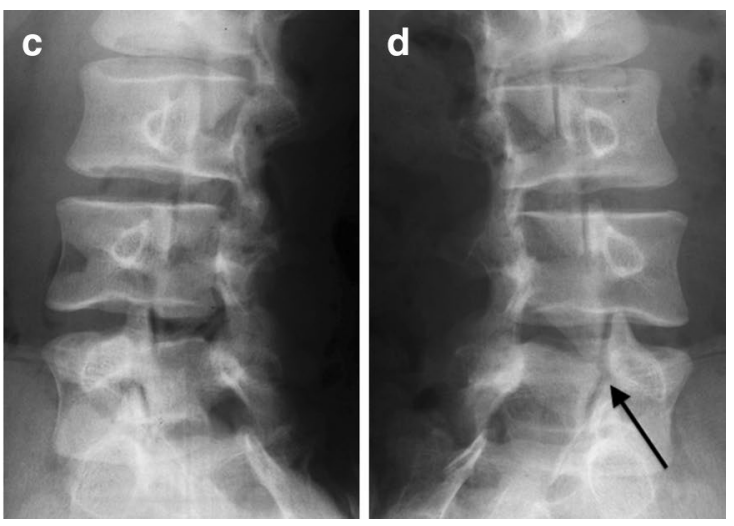

so-called dense pedicle sign (arrow). b No anterolisthesis is seen on lateral view. c,d The pars defect (arrow) is typical on left posterior oblique view without pars defect of the contralateral side

unilateral spondylolysis seemed to be uncommon, and the degree of slippage was considered to be minimal. Cases of spondylolisthesis with unilateral spondylolysis accompanying a slippage ranging 3-8 $\mathrm{mm}$ has been reported [54], and Beutler et al. showed unilateral spondylolysis without spondylolisthesis [22].

Upper lumbar vertebrae

Spondylolysis of the upper lumbar spine is considered to be rare, and some authors estimated $0.2-1.5 \%$ prevalence of upper lumbar spondylolysis (Fig. 7) [7, 13-15, 55]. Unilateral spondylolysis is common in the upper lumbar spine. The frequency of lumbar spondylosis of L3 is estimated to be $1-2 \%$, and involvement of L1 and L2 has been thought to be very rare [7]. It is known that the thickness and strength of pars interarticularis decreases in the upper lumbar spine [56], so the rarity has been thought to be 

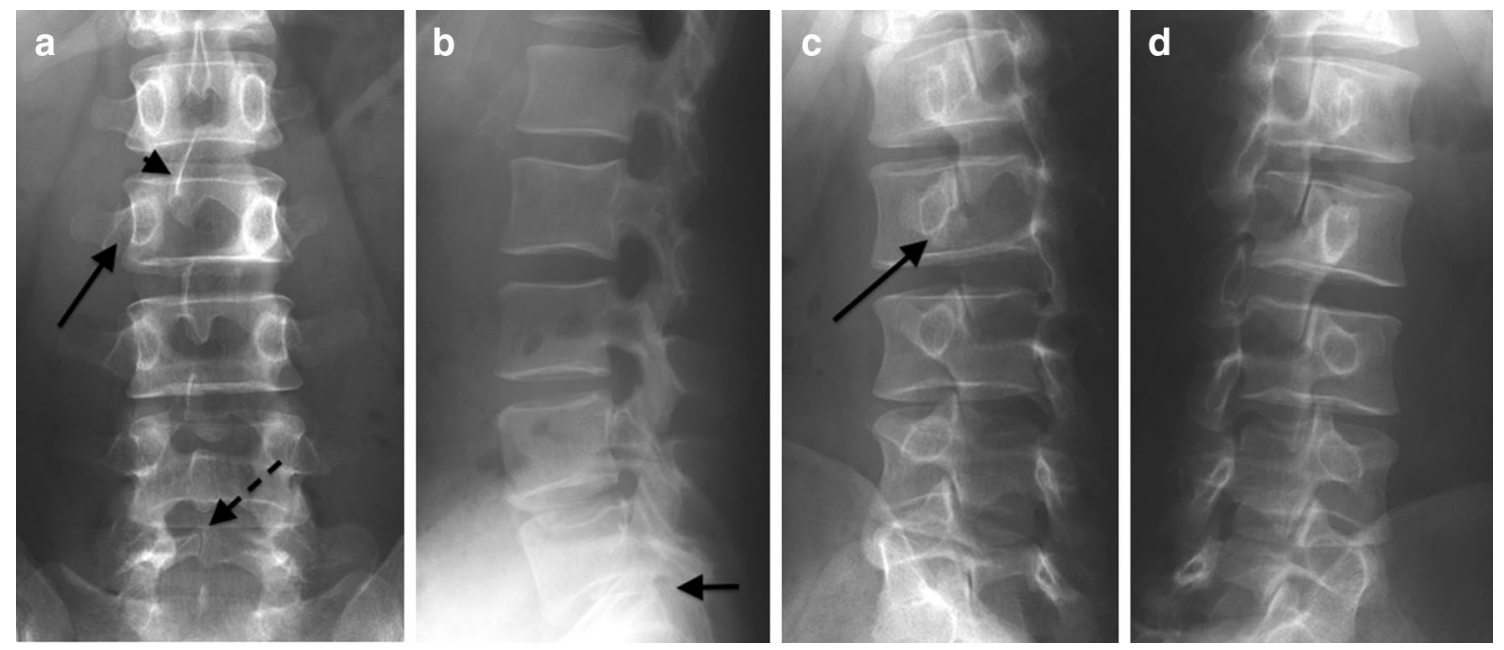

Fig. 7 Spondylosis in the upper lumbar spine (35-year-old male with back pain). Plain radiography of the lumbar spine: a AP view, b lateral view, $\mathbf{c}$ right posterior oblique view, $\mathbf{d}$ left posterior oblique view. a A defect runs obliquely below the pedicle (arrow). The spinous process at L2 is slightly deviated (arrow head). Spina bifida at L5 is

related to lower shearing forces during extension and flexion [7, 27]. The spondylolysis of the upper lumbar spine is often unilateral and the course of the defect is frequently atypical $[6,7]$. Defects orienting obliquely, horizontally or vertically through the pars can be detected by antero-posterior and oblique radiography. Bilateral spondylolysis of the upper lumbar spine is considered to be due to increased mechanical stress to the contralateral side in the presence of unilateral spondylolysis. Because of the high frequency of unilateral spondylolysis, sclerosis and hypertrophy of the contralateral pedicle may be associated. The high ratio of congenital anomaly, such as a spina bifida of the same or nearby affected level has been observed [7, 26].

\section{Natural course}

The natural course seems to vary (Fig. 8). In a recent prospective study of the natural history of spondylolysis and spondylolisthesis, the authors showed that the overall prevalence of spondylolysis during 45 combined years of follow-up was $4.5 \%$ [22]. One healed among 8 unilateral spondylolyses. No slippage was found in the group of unilateral spondylolyses over the course of the study. Three of the bilateral spondylolyses healed, and spondylolisthesis did not develop in four subjects, with average slip of $11 \%$. Slip progression was slow. They concluded that a unilateral pars defect did not cause spondylolisthesis. A bilateral pars defect followed a clinical course similar that of the general population. Slow slip progression with each decade to the age of 50 years was observed, and a bilateral pars defect also noted (dotted arrow). b Degenerative change of L4/5 is observed on lateral view. c, $\mathbf{d}$ Typical pars defect with minimal sclerosis of the right pedicle is seen on right oblique lateral view (arrow). No pars defect is seen on the left side

might develop symptomatic progression in only a small percentage of subjects [22]. It is considered that the progression of spondylolisthesis seemed to be slow in our follow-up cases, but the follow-up cases were few and many subjects of atypical defects in our institution were lost to follow-up evaluations because they were asymptomatic or with no disability. In a case of retroisthmic cleft followed for 6 years with conservative treatment [42], a diminished cleft was reported. No follow-up study of pedicular cleft followed conservatively has been observed.

\section{Conclusions}

The imaging features of spondylolytic spondylolisthesis are various, depending on the stage, type and location. The typical isthmic spondylolysis can be diagnosed on plain radiography, and MR imaging, especially a fluid-sensitive T2-weighted image, is useful in the detection of bone marrow edema of the pars interarticularis at an early stage of spondylolysis, since fracture healing may not occur before the presence of bone marrow edema and a bony fusion can be noted by conservative treatment without surgical intervention. Atypical isthmic spondylolysis is rare and a bone defect having the vertical inferolateral portion and the horizontal superomedial portion is seen in the majority of the patients. Atypical locations of spondylolysis, such as retrosomatic and retroisthmic cleft are rarely seen. Unilateral spondylolysis is uncommon and it occurred in the lower lumbar spine and may be symptomatic. Spondylolysis in the upper lumbar spine is often unilateral and associated 

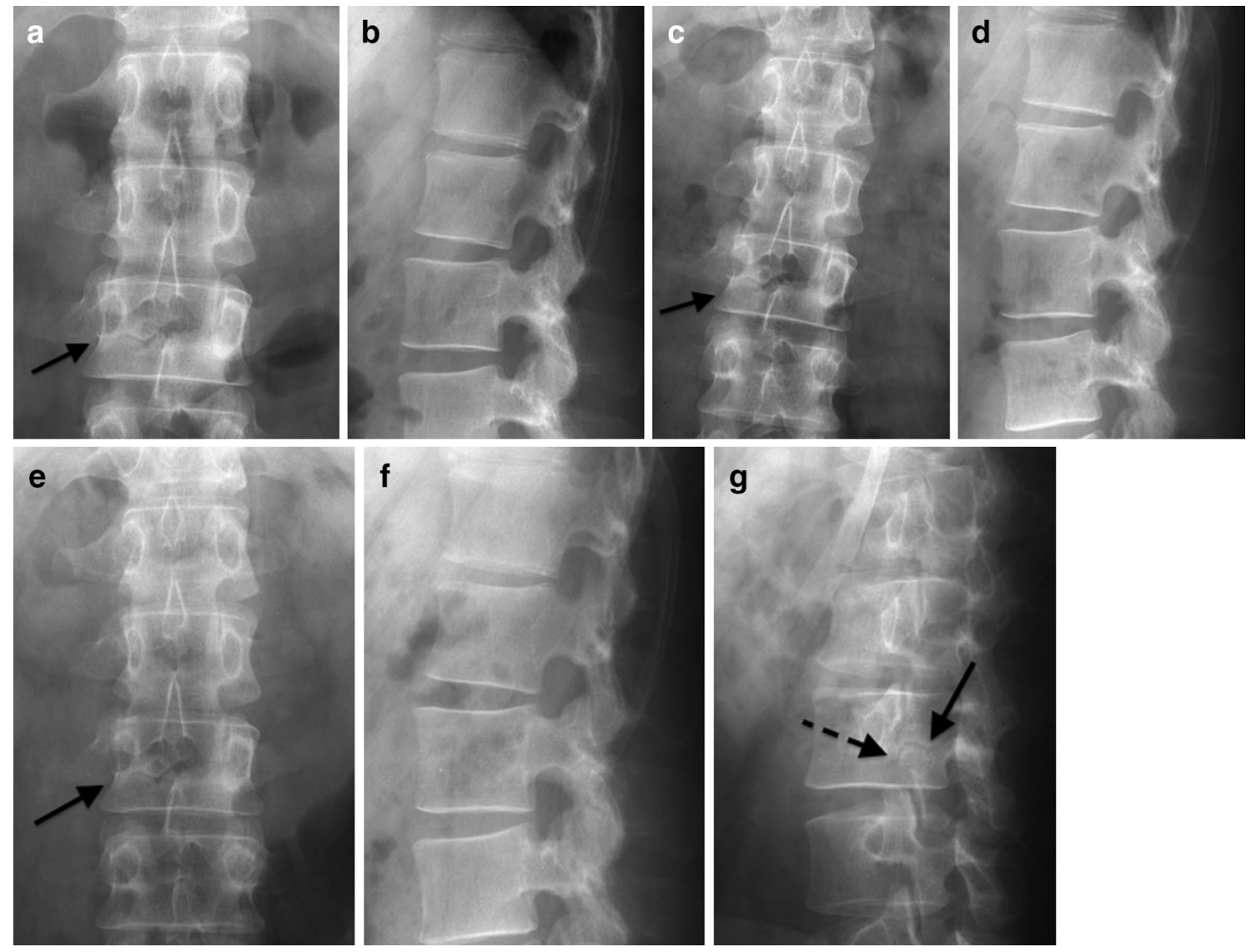

Fig. 8 Atypical and unilateral spondylolysis (23-year-old male with back pain at the time of the initial evaluation). Initial plain radiography of lumbar spine. a AP view, b lateral view. a A defect runs transversally below the right pedicle of L3 (arrow). b No slippage is seen on lateral view. Plain radiography of the lumbar spine obtained 2 years later. c AP view, d lateral view. The defect (arrow) is also

with congenital vertebral anomalies. The natural history is also various with different time courses of evolution and involution.

Conflict of interest The authors declare that they have no conflict of interest.

\section{References}

1. Leone A, Cianfoni A, Cerase A, Magarelli N, Bonomo L. Lumbar spondylosis: a review. Skeletal Radiol. 2011;40:683-700.

2. Brooks BK, Southam SL, Mlady GW, Logan J, Rosett M. Lumbar spine spondylolysis in the adult population: using computed tomography to evaluate the possibility of adult onset lumbar spondylosis as a cause of back pain. Skeletal Radiol. 2010;39:669-73.

3. Sakai T, Sairyo K, Takano S, Nishitani H, Yasui N. Incidence at the age 20 and 29 ages of lumbar spondylolysis in the general population in Japan based on multidetector computed tomography scans from two thousand subjects. Spine. 2009;34(21):2346-50. seen at the same site without increasing gap or displacement. Plain radiography of the lumbar spine obtained 16 years later. e AP view, f lateral view, $\mathbf{g}$ right posterior oblique view. e,f No significant interval change of the defect of L2 has been observed during the 16-year follow-up. g The defect has horizontal (arrow) and vertical (dotted arrow) components, consistent with atypical pars defect

4. Wistle LL. Spondylolisthesis: classification and etiology. In: Symposium on the spine. American Academy of Orthopedic Surgeons. St. Louis: Mosby; 1969. p. 143-67.

5. Kalichman L, Kim DH, Li L, Guermazi A, Berkin V, Hunter J. Spondylolysis and spondylolisthesis: prevalence and association with low back pain in the adult community-based population. Spine. 2009;34:199-205.

6. Nathan H. Spondylolysis. J Bone Jt Surg (Am). 1959;41:303-20.

7. Lee JH, Ehara S, Tamakawa Y, Shimamura T. Spondylolysis of the upper lumbar spine: radiological features. Clin Imag. 1999;23:389-93.

8. Wick L, et al. Retroisthmic cleft: a stress fracture of the lamina. Skeletal Radiol. 2000;29:162-4.

9. Johansen JG, Hemminghytt S, Haughton VM. Retrosomatic clefts: computed tomographic appearance. Radiology. 1983;148:447-8.

10. Guillodo Y, Botton E, Saraux A, LeGoff P. Contralateral spondylolysis and fracture of the lumbar pedicle in an elite female gymnast: a case report. Spine. 2000;25:2541-3.

11. Park JS, Moon SK, Jin W, Ryu KN. Unilateral lumbar spondylosis on radiography and MRI: emphasis on morphologic differences according to involved segment. AJR. 2010;194:207-15. 
12. Rankine JJ, Dickson RA. Unilateral spondylolysis and the presence of facet joint tropism. Spine. 2010;21:E1111-4.

13. Lowe J, Libson E, Ziv I, Nyska M, Floman Y, Bloom RA, Robin GC. Spondylolysis in the upper lumbar spine. A study of 32 patients. J Bone Jt Surg (Br). 1987;69:260-6.

14. Ravichandran G. Upper lumbar spondylolysis. Int Orthop. 1981;5:31-5.

15. Jackson AM, Kirwan EO, Sullivan MF. Lytic spondylolisthesis above lumbosacral spine. Spine. 1978;3:260-6.

16. Wiltse LL, et al. Classification of spondylolysis and spondylolisthesis. Clin Orthop. 1976;177:23-9.

17. Marchetti PG, Bartolozzi P Spondylolisthesis: classification of spondylolisthesis as a guideline for treatment. In: The textbook of spinal surgery, $2^{\text {nd }}$ Ed, Philadelphia: Lippincott-Raven; 1997. p. 1211-54.

18. Hammerberg KW. New concepts on the pathogenesis and classification of spondylolisthesis. Spine. 2005;65:S4-11.

19. Logroscino G, Mazzau O, Aulisa G, Pola E, Aulisa L. Spondylolysis and spondylolisthesis in the pediatric and adolescent population. Child's Nerv Syst. 2001;17:644-55.

20. Ward CV, Latimer B, Alander DH, Parker J, Ronan JA, Holden $\mathrm{AD}$, Sanders C. Radiographic assessment of lumbar facet distance spacing and spondylolysis. Spine. 2007;32:E35-8.

21. Masharawi YM, Alperovitch-Najenson D, Steinberg N, Dan G, Peleg S, Rothschild B, Salame K, Hershkovitz I. Lumbar facet orientation in spondylolysis: a skeletal study. Spine. 2007;32:E176-80.

22. Beutler WJ, Fredrickson BE, Murtland A, Sweeney CA, Grant WD, Baker D. The natural history of spondylolysis and spondylolisthesis. 45-year follow-up evaluation. Spine. 2003;28:1027-35.

23. Ikata T, Miyake R, Katoh S, Morita Y, Murase M. Pathogenesis of sports-related spondylolisthesis in adolescents: radiographic and magnetic resonance imaging study. Am J Sports Med. 1996;24:94-8.

24. Floman Y. Progression of lumbosacral isthmic spondylolisthesis in adults. Spine. 2000;25:342-7.

25. Mihara H, Onari K, Cheng BC, David SM, Zdeblick TA. The biomechanical effect of spondylolysis and its treatment. Spine. 2003;28:235-8.

26. Fredrickson BE, Baker D, McHolick WJ, Yuan HA, Lubicky JP. The natural history of spondylolysis, and spondylolisthesis in children and adolescents. JBJS Am. 1984;66:699-707.

27. Farfan HF, Osteria V, Lamy C. The mechanical etiology of spondylolysis and spondylolisthesis. Clin Orthop. 1976;117:40-55.

28. Sairyo K, Katoh S, Ikata T, Fujii K, Kajiura K, Goel VK, Murakami RI. The pathomechanism of isthmic lumbar spondylolisthesis. Spine J. 2001;1:171-5.

29. Floman Y. Progression of lumbosacral isthmic spondylolisthesis in adults. Spine. 2000;25:342-7.

30. McGregor AH, Anderton L, Gedroyc WM, Johnson J, Hughes SP. The use of interventional open MRI to assess the kinematics of lumbar spine in a patient with spondylolisthesis. Spine. 2002;27:1582-6.

31. Axelsson P, Johnson R, Stromqvist B. Is there increased intervertebral mobility in isthmic adult spondylolisthesis? A matched comparative study using roentgen stereophotogrammetry. Spine. 2000;25:1710-3.

32. Szypryt EP, Twining P, Mulholand RC, Worthington BS. The prevalence of disc degeneration associated with neural arch defects of the lumbar spine assessed by magnetic resonance imaging. Spine. 1989;14:977-81.

33. Dunn A, Campbell R, Mayor P, Rees D. Radiological findings and healing patterns of incomplete stress fractures of the pars interarticularis. Skeletal Radiol. 2008;37(5):443-50.

34. Sairyo K, Katoh S, Tanaka Y, Terai T, Yasui Y, Goel VK, Masuda A, Vadapalli S, Biyani A, Ebraheim N. MRI signal changes of the pedicle as an indicator for early diagnosis of spondylolysis in children and adolescents. Spine. 2006;31:206-11.

35. Campbell RS, Grainger AJ, Hide IG, Papastefanous S, Greenough CG. Juvenile spondylolysis: a comparative analysis of CT. SPECT MRI. Skeletal Radiol. 2005;34(5):63-73.

36. Hollenberg GM, Beattie PF, Meyers SP, Weinberg EP, Adams MJ. Stress reactions of the lumbar pars interarticularis: the development of a new classification system. Spine. 2002;27:181-6.

37. Ulmer JL, Mathews VP, Elster AD, Mark LP, Daniels DL, Mueller W. MR imaging of lumbar spondylolysis: the importance of ancillary observations. Am J Roentgenol. 1997;169:233-9.

38. Sherif H, Mahfouz AE. Epidural fat interposition between dura mater and spinous process: a new sign of spondylolysis on MR imaging of the lumbar spine. Eur Radiol. 2004;14(6):970-3.

39. Fujii K, Katoh S, Sairyo K, Ikata T, Yasui Y. Union of defects in the pars articularis of the lumbar spine in children and adolescents: the radiological outcome after conservative treatment. J Bone Jt Surg Br. 2004;86(2):225-31.

40. Wiltse LL. The etiology of spondylolisthesis. J Bone Jt Surg (Am). 1962;44:539-60.

41. Wick L, Kaim A, Bougantz G. Retroisthmic cleft: a stress fracture of the lamina. Skeletal Radiol. 2000;29:162-4.

42. Sairyo K, Katoh S, Sasa T, Yasui N, Goel VK, Vadapalli S, Masuda A, Biyani A, Ebraheim N. Athletes with unilateral spondylolysis are at risk of stress fracture at the contralateral pedicle and pars interarticularis: a clinical and biomechanical study. Am J Sports Med. 2005;33:583-90.

43. Sairyo K, Katoh S, Komatsubara S, Terai T, Yasui N, Goel VK, Vadapalli A, Biyani A, Ebraheim N Spondylolysis fracture angle in children and adolescents on $\mathrm{CT}$ indicates the fracture-producing force vector: a biomechanical rationale. Int J Spine Surg; 2011.p.22.

44. Wortzman G, Steinharrdt MI. Congenital absent lumbar pedicle: a reappraisal. Radiol. 1984;152:713-8.

45. Johansen JG, Hemminghytt S, Haughton VM. CT appearance of the retroisthmic cleft. AJNR. 1984;5:835-6.

46. Abraham T, Holder L, Silberstein C. The retroisthmic cleft. Scintigraphic appearance and clinical relevance in the patients with low back pain. Clin Nucl Med. 1997;22:161-5.

47. Horner CW, Haughton VM. MR appearance of the retroisthmic cleft. AJNR. 1996;17:397-8.

48. Garber JE, Wright AM. Unilateral spondylolysis and contralateral pedicle fracture. Spine. 1986;11:63-6.

49. Bensaid AH, Dietemann JL, Kastler B, Gangi A, Jeung MY, Wackenheim A. Neurofibromatosis with dural ectasia and bilateral symmetrical pedicular clefts. Neuroradiol. 1992;34:107-9.

50. Amari R, Sakai T, Katoh S, Sairyo K, Higashino K, Tabuchi K, Yaui N. Fresh stress fractures of lumbar pedicles in an adolescent male ballet dancer: case report and literature review. Arch Orthop Trauma Surg. 2009;129:397-401.

51. Doita M, Shimomura T, Nishida K, Maeno K, Fujioka H, Kurosawa M. Bilateral pedicle stress fracture in patients with lumbar spinal stenosis. J Spinal Disord Tech. 2008;21:531-4.

52. Ha KY, Kim YH. Bilateral pedicle stress fracture after instrumented posterolateral lumbar fusion: a case report. Spine. 2003;28:E158-60.

53. Merbs CF. Asymmetrical spondylolysis. Am J Phys Anthropol. 2002;119:156-74.

54. Einstein S, Spondylolysis A. Skeletal investigation of two population groups. J Bone Jt Surg Br. 1978;60:4988-94.

55. Cryron BM, Hutton WC, Troup JDG. Spondylolytic fractures. J Bone Jt Surg Br. 1976;58:462-6.

56. Troup JDG. Mechanical factors in spondylolisthesis and spondylolysis. Clin Orthop. 1976;117:59-67. 